\title{
Thermodynamics and Excitations of Condensed Polaritons in Disordered Microcavities
}

\author{
F. M. Marchetti, ${ }^{1}$ J. Keeling, ${ }^{1}$ M. H. Szymańska, ${ }^{2}$ and P. B. Littlewood ${ }^{1}$ \\ ${ }^{1}$ Cavendish Laboratory, University of Cambridge, Madingley Road, Cambridge CB3 OHE, United Kingdom \\ ${ }^{2}$ Clarendon Laboratory, Department of Physics, University of Oxford, Parks Road, Oxford, OX1 3PU, United Kingdom
}

(Received 16 September 2005; published 17 February 2006)

\begin{abstract}
We study the thermodynamic condensation of microcavity polaritons using a realistic model of disorder in semiconductor quantum wells. This approach correctly describes the polariton inhomogeneous broadening in the low density limit, and treats scattering by disorder to all orders in the condensed regime. While the weak disorder changes the thermodynamic properties of the transition little, the effects of disorder in the condensed state are prominent in the excitations and can be seen in resonant Rayleigh scattering.
\end{abstract}

\section{DOI: 10.1103/PhysRevLett.96.066405}

Considerable effort has been recently devoted to the realization of a Bose-Einstein condensate of polaritons in III-V and II-VI semiconductor microcavities [1-3]. The very light mass of these composite bosonic particles promises relatively high transition temperatures, establishing these systems as ideal candidates for observing condensation. A significant challenge to the realization of an equilibrium condensate might be represented by the short polariton lifetime (caused by the finite quality of cavity mirrors) and by the suppression of thermalization processes by acoustic phonons at small momenta - the "bottleneck effect." However, very recent developments have suggested that, by positively detuning the cavity energy above the exciton energy, and by increasing the nonresonant pump power to increase particle-particle scattering, thermalization can be dramatically amplified [4]. While unambiguous evidence for equilibrium condensation still remains uncertain, much progress has been achieved in this direction. This includes the observation of a nonlinear threshold behavior in the emission intensity under nonresonant pump, the decrease, above threshold, of the second order coherence function [2] together with a characteristic change in the momentum space distribution, and, recently, interference patterns in far-field emission have been measured [3].

Theoretical effort has also been devoted to predicting properties and possible signatures of polariton condensation $[5,6]$. In this Letter we consider how disorder, through the distribution of excitonic energies and oscillator strengths, affects such signatures. Even with the sophisticated growth technologies used in current structures, the presence of interface and alloy disorder induces noticeable effects. Here, we will show that the response under resonant Rayleigh scattering (RRS), the coherent scattering by the disorder of an injected photon into directions other than its original direction, provides a unique probe of the condensed regime. Already in the low density (linear) regime, disorder determines the RRS response $[7,8]$ and the inhomogeneous broadening of the polariton photoluminescence (PL). Using a quantitatively accurate model for the exciton disorder $[9,10]$ that has already been well tested in
PACS numbers: 71.36.+c, 71.45.- d, 78.35.+c

the linear regime of excitation, we investigate the effects of disorder on an equilibrium polariton condensation. We find that the thermodynamic properties of the transition are weakly affected by small disorder, as expected. However, at densities above the threshold expected for condensation, the normal modes supported by the cavity change from the lower and upper polariton modes to new collective excitations [5]. Accordingly, the response of the condensate to an additional RRS probe also changes. In noticeable contrast with the noncondensed regime, the frequency-resolved RRS emission from the linear Goldstone mode exists both above and below the chemical potential. Moreover, the spectrum exhibits features directly related to the disordered quasiparticle spectrum. Here, in analogy with the BCS theory, the quasiparticles are given by "particle-hole" excitations (i.e., bound excitons) coupled to the coherent photon field via the random, disorder dependent, oscillator strength.

The linear response of a resonantly pumped polaritonic system to an external disorder potential has been recently studied in [11]. In that work, in contrast with the case analyzed here, the coherence of the system is driven by the pump, and moreover the effect of disorder is included at a perturbative level.

The influence of quantum well disorder on excitonic energies and oscillator strengths has been much studied in the last two decades [for an exhaustive discussion see, e.g., [9] and references therein]. Here, we assume the disorder to be correlated on a length scale $\ell_{c}$ larger than the exciton Bohr radius $a_{x}=\epsilon / e^{2} \mu_{r}$, where $\mu_{r}$ is the reduced mass (henceforth we will set $\hbar=1$ ). Accordingly, we factorize the in-plane relative and center of mass coordinates, $\Psi_{\alpha}\left(\mathbf{r}_{e}, \mathbf{r}_{h}\right) \simeq \varphi_{1 s}(r) \Phi_{\alpha}(\mathbf{R})$, and focus on the excitonic center of mass motion,

$$
\left[-\frac{\nabla_{\mathbf{R}}^{2}}{2 m_{x}}+V(\mathbf{R})\right] \Phi_{\alpha}(\mathbf{R})=\varepsilon_{\alpha} \Phi_{\alpha}(\mathbf{R})
$$

where the energies are measured with respect to the exciton band edge $E_{x}$, i.e., the band gap minus the exciton Rydberg $\mathcal{R}_{x}=\left(2 \mu_{r} a_{x}^{2}\right)^{-1}$. The effective disorder potential $V(\mathbf{R})$ 
represents the microscopic structural disorder averaged over the electron-hole motion [10] and can be approximated, e.g., with a Gaussian noise, $\left\langle V(\mathbf{R}) V\left(\mathbf{R}^{\prime}\right)\right\rangle=$ $\left(\sigma^{2} \ell_{c}^{2} / L^{2}\right) \sum_{\mathbf{q}}^{1 / \ell_{c}} e^{i \mathbf{q} \cdot\left(\mathbf{R}-\mathbf{R}^{\prime}\right)}$, where $L^{2}$ is the quantization area.

Even though, in two-dimensional noninteracting systems, all states are localized by the disorder potential, the character of the excitonic wave function changes significantly from below to above the band edge $E_{x}$. The coupling strength of an exciton to light changes accordingly. For dipole-allowed transitions, the exciton oscillator strength $g_{\alpha, \mathbf{p}}$ is proportional to the probability amplitude $\varphi_{1 s}(0) \Phi_{\alpha, \mathbf{p}}$ of finding an electron and a hole at the same position and with center of mass momentum equal to the photon momentum $\mathbf{p}[9,10]$,

$$
g_{\alpha, \mathbf{p}}=e d_{a b} \sqrt{\frac{2 \pi \omega_{\mathbf{p}}}{\epsilon L_{w}}} \varphi_{1 s}(0) \Phi_{\alpha, \mathbf{p}},
$$

where $d_{a b}$ is the dipole matrix element. Here, we solve Eq. (1) numerically on a grid of $120 \times 120$ points (for which convergence is reached) for a system of size $L=$ $1 \mu \mathrm{m}, \sigma=2 \mathrm{meV}$, and $\ell_{c}=166 \AA$. From the evaluated eigenvalues $\varepsilon_{\alpha}$ and eigenstates $\Phi_{\alpha, \mathbf{p}}$, we can derive the excitonic density of states $\operatorname{DoS}(\varepsilon)$, the coupling strength $g_{\alpha, \mathbf{p}}$, and its squared average (Fig. 1):

$$
g^{2}(\varepsilon,|\mathbf{p}|)=\frac{1}{\mathcal{D o S}(\varepsilon)}\left\langle\sum_{\alpha}\left|g_{\alpha, \mathbf{p}}\right|^{2} \delta\left(\varepsilon-\varepsilon_{\alpha}\right)\right\rangle,
$$

where $\langle\ldots\rangle$ is the average over different disorder realizations. This quantity is related to the excitonic optical density by $D(\varepsilon)=\mathcal{D o S}(\varepsilon) g^{2}(\varepsilon, 0)$.

We now consider the following Hamiltonian describing excitons with random energies $\varepsilon_{\alpha}$ dipole coupled via $g_{\alpha, \mathbf{p}}$ to the cavity field $\psi_{\mathbf{p}}$ :

$$
\begin{aligned}
\hat{H}= & \sum_{\alpha} \frac{\varepsilon_{\alpha}}{2}\left(b_{\alpha}^{\dagger} b_{\alpha}+a_{\alpha} a_{\alpha}^{\dagger}\right)+\sum_{\mathbf{p}} \omega_{\mathbf{p}} \psi_{\mathbf{p}}^{\dagger} \psi_{\mathbf{p}} \\
& +\frac{1}{\sqrt{L^{2}}} \sum_{\alpha} \sum_{\mathbf{p}}\left(g_{\alpha, \mathbf{p}} \psi_{\mathbf{p}} b_{\alpha}^{\dagger} a_{\alpha}+\text { H.c. }\right) .
\end{aligned}
$$

Interactions are approximated by exclusion, so each exciton level $\varepsilon_{\alpha}$ is modeled by an electron-hole pair $a$ and $b$, with the total occupation restricted to one, i.e., $b_{\alpha}^{\dagger} b_{\alpha}+$ $a_{\alpha}^{\dagger} a_{\alpha}=1$. For the thermodynamical properties of this model (e.g., the critical temperature), this is a good assumption at low enough densities, where only the strongly localized (Lifshitz) states in the tail are populated. The dispersion for photons in a microcavity of width $L_{w}$ is approximated as parabolic, $\omega_{\mathbf{p}} \simeq \omega_{0}+\mathbf{p}^{2} / 2 m_{\mathrm{ph}}$, where $\omega_{0}=2 \pi c / L_{w} \sqrt{\epsilon}$ and $m_{\mathrm{ph}}=2 \pi \sqrt{\epsilon} / c L_{w}$. It is convenient to rescale the exciton-light coupling $g_{\alpha, \mathbf{p}}$ according to $g_{\alpha, \mathbf{p}} \mapsto g_{\alpha, \mathbf{p}} \sqrt{\mathcal{R}_{x} m_{x} / 2 \pi}$, where $N=\mathcal{R}_{x} L^{2} m_{x} / 2 \pi$ is the inverse level spacing measured in units of the excitonic Rydberg energy. This corresponds to measuring the density
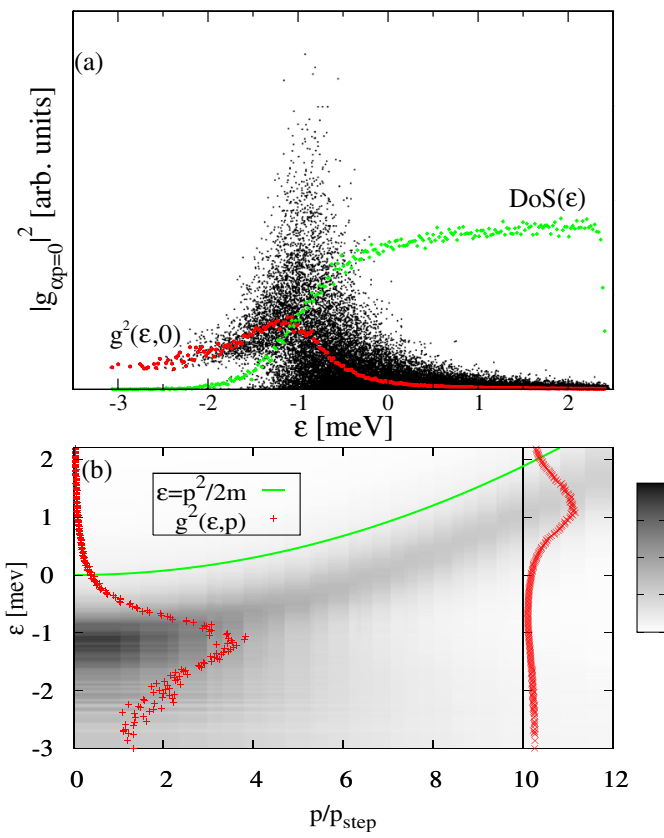

FIG. 1 (color online). (a) Plot of the squared coupling strength $\left|g_{\alpha, \mathbf{p}}\right|^{2}$ vs energy (160 realizations of disorder) for $\mathbf{p}=0$; (b) contour plot of the squared averaged oscillator strength $g^{2}(\varepsilon,|\mathbf{p}|)$ vs energy and momentum. The resolution in momentum, $p_{\text {step }}=2 \pi / L=6.3 \times 10^{4} \mathrm{~cm}^{-1}$ corresponds, for a cavity of $\omega_{0}=1.68 \mathrm{eV}$, to an angle of $\theta_{\text {step }}=\tan ^{-1}\left(c p_{\text {step }} / \omega_{0}\right)=36^{\circ}$. The free particle dispersion $|\mathbf{p}|^{2} / 2 m_{x}$ (solid line), and the squared averaged oscillator strength for two values of momenta, $|\mathbf{p}|=0$ and $|\mathbf{p}|=10 p_{\text {step }}\left(\theta=82^{\circ}\right)$ (plus symbols) are explicitly plotted.

of particles in units of the Bohr radius squared. In thermal equilibrium, the total number of excitations, $\hat{N}=$ $\sum_{\alpha}\left(b_{\alpha}^{\dagger} b_{\alpha}+a_{\alpha} a_{\alpha}^{\dagger}\right) / 2+\sum_{\mathbf{p}} \psi_{\mathbf{p}}^{\dagger} \psi_{\mathbf{p}}$, can be fixed by introducing a chemical potential, $\mu$. The dimensionless density $\rho \equiv\langle\hat{N}\rangle / N$ corresponds to $\left(\langle\hat{N}\rangle / L^{2}\right) a_{x}^{2} 4 \pi \mu_{r} / m_{x}$, where $\langle\hat{N}\rangle / L^{2}$ is the density of particles per area.

Making use of standard path integral techniques, the mean-field equations for the static and uniform photon field $\psi$ and for the total number of excitations can be simultaneously solved in a similar way as in Refs. [5,12]. However, in contrast to previous work, we introduce here a realistic description for the excitons in the disordered quantum wells and make use of the energies and coupling strengths evaluated numerically. In addition, the average over different disorder realizations outside the energy interval evaluated numerically [Fig. 1(a)] is taken by extrapolating the numerics: in the low energy Lifshitz tail, we approximate the distribution of $\left|g_{\alpha, 0}\right|^{2}$ with a delta function at its extrapolated mean value $g^{2}(\varepsilon, 0)$, while, in the high energy region, we use the Porter-Thomas distribution $\mathcal{P}\left(x=\left|g_{\alpha, 0}\right|^{2}\right)=\exp [-x /(2 \bar{x})] / \sqrt{2 \pi x} \bar{x}, \quad$ where $\quad \bar{x}=$ $g^{2}(\varepsilon, 0)$. The scale of $\left|g_{\alpha, 0}\right|^{2}$ is set so as to fix the integrated optical density $\int d \varepsilon D(\varepsilon)=\Omega_{\mathrm{R}}^{2} / 4$, where $\Omega_{\mathrm{R}}$ is the measured Rabi splitting. In the resulting mean-field phase diagram (Fig. 2), increasing the density of particles at a 
given temperature, the system goes under a phase transition from a noncondensed phase $(\psi=0)$ to a phase where the polaritons condense in the lowest momentum state $(\psi \neq 0)$.

The incoherent PL spectrum and the RRS response are evaluated by considering fluctuations of the photonic field above the mean-field solution:

$$
\begin{aligned}
\delta \mathcal{S} & \simeq \frac{1}{2 k_{B} T} \sum_{\omega_{h}, \mathbf{p}, \mathbf{q}}\left(\begin{array}{c}
\delta \psi_{\omega_{h}, \mathbf{p}}^{*} \\
\delta \psi_{-\omega_{h},-\mathbf{p}}
\end{array}\right)^{T} \mathcal{G}_{\mathbf{p q}}^{-1}\left(\omega_{h}\right)\left(\begin{array}{c}
\delta \psi_{\omega, \mathbf{q}} \\
\delta \psi_{-\omega_{h},-\mathbf{q}}^{*}
\end{array}\right) \\
\mathcal{G}_{\mathbf{p q}}^{-1}\left(\omega_{h}\right) & =\left(\begin{array}{cc}
K_{\mathbf{p q}}^{(1)}\left(\omega_{h}\right) & K_{\mathbf{p q}}^{(2)}\left(\omega_{h}\right) \\
K_{\mathbf{p q}}^{(2)}\left(\omega_{h}\right) & K_{\mathbf{p q}}^{(1) *}\left(\omega_{h}\right)
\end{array}\right) .
\end{aligned}
$$

Here, when $\omega_{h}=2 \pi k_{B} T h \neq 0$, the matrix elements of the inverse quasiparticle Green's function are given by

$$
\begin{aligned}
& K_{\mathbf{p q}}^{(1)}\left(\omega_{h}\right)= \delta_{\mathbf{p}, \mathbf{q}}\left(i \omega_{h}+\tilde{\omega}_{\mathbf{p}}\right)+\frac{1}{N} \sum_{\alpha} g_{\alpha, \mathbf{p}}^{*} g_{\alpha, \mathbf{q}} \\
& \times\left[i \omega_{h} \tilde{\varepsilon}_{\alpha} / 2-E_{\alpha}^{2}-\left(\tilde{\varepsilon}_{\alpha} / 2\right)^{2}\right] h\left(E_{\alpha}\right) \\
& K_{\mathbf{p q}}^{(2)}\left(\omega_{h}\right)=\frac{\psi^{2}}{N} \frac{1}{N} \sum_{\alpha}\left|g_{\alpha, 0}\right|^{2} g_{\alpha, \mathbf{p}}^{*} g_{\alpha, \mathbf{q}} h\left(E_{\alpha}\right),
\end{aligned}
$$

where $h(x)=\tanh \left(x / k_{B} T\right) /\left[x\left(\omega_{h}^{2}+4 x^{2}\right)\right]$,

$$
E_{\alpha}=\sqrt{\left(\tilde{\varepsilon}_{\alpha} / 2\right)^{2}+\left|g_{\alpha, 0}\right|^{2} \psi^{2} / N}
$$

is the energy of an exciton in a coherent field, $\tilde{\varepsilon}_{\alpha}=\varepsilon_{\alpha}-$ $\mu$, and $\tilde{\omega}_{\mathbf{p}}=\omega_{\mathbf{p}}-\mu$. The quasiparticle Green's function can be decomposed into (momentum) diagonal and offdiagonal contributions, $K_{\mathbf{p q}}^{(1,2)}=K_{\mathbf{p q}}^{(1,2) d} \delta_{\mathbf{p q}}+K_{\mathbf{p q}}^{(1,2) o}$. The off-diagonal terms, as they break translational invariance and therefore have a zero average over different disorder realizations, can be neglected when evaluating the incoherent PL intensity [13], $P(\omega, \mathbf{p})=n_{B}(\omega) W(\omega, \mathbf{p})$, where

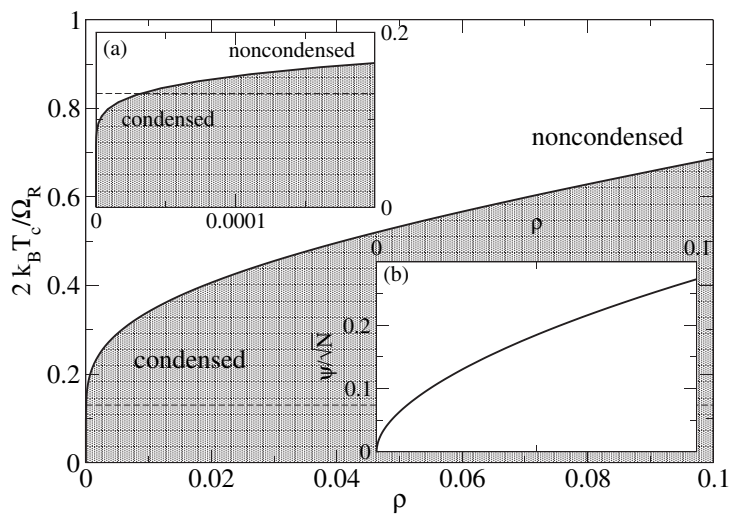

FIG. 2. Mean-field phase diagram for the dimensionless critical temperature $2 T_{c} k_{B} / \Omega_{\mathrm{R}}$ vs the dimensionless density $\rho$ for effective zero detuning $\omega_{0}-E_{x}=-0.94 \mathrm{meV}$ and $\Omega_{\mathrm{R}}=$ $26 \mathrm{meV}$. A detail of the low density region is shown in the inset (a), while the plot of the mean-field order parameter $\psi / \sqrt{N}$ vs the density for $T k_{B}=20 \mathrm{~K}\left(2 T k_{B} / \Omega_{\mathrm{R}}=0.13\right)$ is shown in the inset (b).
$n_{B}(\omega)$ is the Bose occupation factor and

$$
W(\omega, \mathbf{p})=\left.2 \operatorname{Im} G_{\mathbf{p p}}^{11}\left(\omega_{h}\right)\right|_{i \omega_{h}=-\omega-i \eta}
$$

is the spectral weight. However, allowing the normal modes supported by the cavity to scatter via their excitonic component in directions different from the incoming one, these terms are essential in finding the RRS intensity:

$$
I_{\mathbf{p q}}(\omega)=\left.\left|G_{\mathbf{p q}}^{11}\left(\omega_{h}\right)\right|^{2}\right|_{i \omega_{h}=-\omega-i \eta} \simeq F_{\mathbf{p}} S_{\mathbf{p q}} F_{\mathbf{q}} .
$$

Here, the filter function $F_{\mathbf{p}}=\left.|| K_{\mathbf{p p}}^{(1)}\right|^{2}-\left.\left[K_{\mathbf{p p}}^{(2)}\right]^{2}\right|^{-2}$ describes the propagation of the injected and detected photons via the normal modes supported by the cavity, while the scattering function $S_{\mathbf{p q}}$

$$
\begin{aligned}
S_{\mathbf{p q}}= & \mid K_{\mathbf{p q}}^{(1) o} K_{\mathbf{p p}}^{(1) *} K_{\mathbf{q q}}^{(1) *}+K_{\mathbf{q p}}^{(1) O *} K_{\mathbf{p p}}^{(2)} K_{\mathbf{q q}}^{(2)} \\
& -\left.K_{\mathbf{p q}}^{(2) o}\left[K_{\mathbf{p p}}^{(2)} K_{\mathbf{q q}}^{(1) *}+K_{\mathbf{p p}}^{(1) *} K_{\mathbf{q q}}^{(2)}\right]\right|^{2}
\end{aligned}
$$

describes the probability to scatter, via the excitonic component, from the injected momentum $\mathbf{p}$ to the detected one q. The incoherent PL $P(\omega, \mathbf{p})$ and spectral weight $W(\omega, \mathbf{p})$, as also the disorder averaged RRS response $\left\langle I_{\mathbf{p q}}(\omega)\right\rangle$, are shown in Fig. 3 for two values of the density at a fixed temperature, showing both the noncondensed [Figs. 3(a), 3(c), and 3(e)] and condensed case [Figs. 3(b), 3(d), and 3(f)].

The RRS signal reflects many of the features already present in the spectral weight. The similarity is due to the

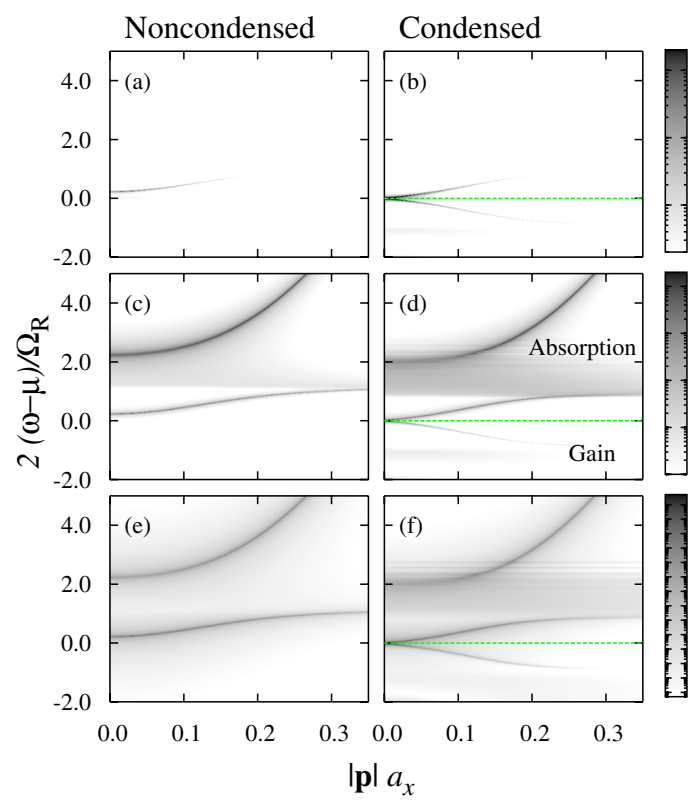

FIG. 3 (color online). Contour plot of the incoherent PL $P(\omega, \mathbf{p})(\mathrm{a}),(\mathrm{b})$, the spectral weight $W(\omega, \mathbf{p})(\mathrm{c}),(\mathrm{d})$, and the disorder averaged RRS intensity $\left\langle I_{\mathbf{p q}}(\omega)\right\rangle$ for $|\mathbf{p}|=|\mathbf{q}|$ (e), (f) vs the rescaled energy $2(\omega-\mu) / \Omega_{\mathrm{R}}$ and the dimensionless momentum $|\mathbf{p}| a_{x}$, for $\omega_{0}-E_{x}=-0.94 \mathrm{meV}, \Omega_{\mathrm{R}}=26 \mathrm{meV}$, $k_{B} T=20 \mathrm{~K}:$ noncondensed (left column) $(\rho \simeq 0, \mu=$ $-16.5 \mathrm{meV})$ and condensed (right column) $\left(\rho=3.6 \times 10^{-3}\right.$, $\mu=-11.3 \mathrm{meV})$. 
filter functions, which limit the RRS response to the normal modes supported by the cavity and are responsible for the ring-shaped emission observed in experiments $[7,8]$. When uncondensed $(\psi \rightarrow 0), K^{(2)}=0$, the filter terms $\left|K^{(1) d}\right|^{-2}$ coincide with the squared polariton Green's function and the scattering term $\left|K^{(1) o}\right|^{2}$ gives the probability for an exciton to scatter from $\mathbf{p}$ to $\mathbf{q}$. At ultralow densities, the model used here is equivalent to that used in Ref. [13] and the RRS response is in agreement with that of Refs. $[14,15]$. However, when condensed, the polariton modes are replaced by new collective modes, the lower polariton becomes a linear Goldstone mode, and two new branches appear below the chemical potential, which are seen as gain in the spectral weight. These changes are also seen in the RRS response, including RRS response at energies below the chemical potential [Fig. 3(f)].

These noticeable changes could be observed in both PL emission, the product of the spectral weight times the Bose occupation factor $n_{B}(\omega)$, and the RRS response. However, because of the occupation factor, PL emission from above the chemical potential is exponentially suppressed. In addition, the PL as it is plotted in Fig. 3 excludes the emission from the condensate, which in experiments might obscure these features. In contrast, RRS response is not weighted by occupation, but instead by the scattering function, $S_{\mathbf{p q}}$, which depends on the modulus squared of the Green's function for an exciton in a coherent field. As a consequence, RRS represents a unique probe for observing condensation in polariton microcavities.

Using the full distribution of oscillator strengths has observable effects in the condensed state and is vital in our treatment. When uncondensed, while the spectral weight depends only on the excitonic optical density (and thus on the average of $\left|g_{\alpha, 0}\right|^{2}$ ), the RRS depends also on $\left|g_{\alpha, 0}\right|^{4}$. This determines a sharper energy dependence in RRS than in optical density. However, when condensed, both optical responses are determined by the full distribution of oscillator strengths, rather than only the mean squared oscillator strength and its mean fourth power. This is because the energy $E_{\alpha}$ of an exciton in the presence of a coherent field leads to a dependence on the full distribution of $\left|g_{\alpha, 0}\right|$. The density of states of these excitonic quasiparticles can be directly seen in the spectral weight between the upper and lower polariton modes. With a constant $g$, the minimum of $E_{\alpha}$ is sharp, occurring for excitons where $\tilde{\varepsilon}_{\alpha}=0$. In contrast, with the distribution of $\left|g_{\alpha, 0}\right|$ used here, the minimum of $E_{\alpha}$ occurs for states where there is first a finite probability of having negligible coupling to light. Below this energy, other than the linear mode, there is a suppression of the spectral weight. The energy dependence of the distribution of coupling strengths is also important, and is responsible for the decrease of polariton splitting at higher densities. As the chemical potential increases, the excitons with energies close to the chemical potential couple less strongly to light, and the effective splitting decreases.
The treatment in this Letter considers only disorder acting on the excitons; it has been suggested $[8,16]$ that disorder of the mirrors, acting on the photons might also be relevant. The inclusion of such disorder can be expected to modify the scattering term, but the filter functions are expected to remain unaffected.

To conclude, we have described how polariton condensation in semiconductor microcavities can be investigated by RRS. By making use of a realistic model of disorder in quantum wells, we identified the changes which can be observed in the RRS spectrum when, with increasing density, the system, still in the strong coupling regime, crosses the phase boundary to a condensed state. Because RRS, in contrast to PL, is not weighted by the Bose factor, and because RRS does not contain a strong signal at the condensate frequency, which could obscure the subtle features in PL, RRS provides a promising probe of polariton condensation in semiconductor microcavities.

We are grateful to B.D. Simons, Le Si Dang, J. Kasprzak, and W. Langbein for suggestions and useful discussions. F. M. M. and M. H. S. would like to acknowledge the financial support of EPSRC. This work is supported by the EU Network "Photon mediated phenomena in semiconductor nanostructures" HPRN-CT-2002-00298.

[1] Le Si Dang et al., Phys. Rev. Lett. 81, 3920 (1998).

[2] H. Deng et al., Science 298, 199 (2002); H. Deng et al., Proc. Natl. Acad. Sci. U.S.A. 100, 15318 (2003).

[3] M. Richard et al., Phys. Rev. Lett. 94, 187401 (2005).

[4] Le Si Dang, J. Kasprzak, H. Deng (private communication).

[5] J. Keeling et al., Phys. Rev. Lett. 93, 226403 (2004); Phys. Rev. B 72, 115320 (2005).

[6] V. Savona et al., Phase Transit. 68, 169 (1999); F. P. Laussy et al., Phys. Rev. Lett. 93, 016402 (2004); A. Kavokin and G. Malpuech, Cavity Polaritons, in Thin Films and Nanostructures Vol. 32 (Elsevier, New York, 2003).

[7] T. Freixanet et al., Phys. Rev. B 60, R8509 (1999).

[8] W. Langbein and J. M. Hvam, Phys. Rev. Lett. 88, 047401 (2002).

[9] E. Runge, Solid State Physics, Excitons in Semiconductors Nanostructures Vol. 57 (Academic, New York, 2002).

[10] R. Zimmermann, F. Grosse, and E. Runge, Pure Appl. Chem. 69, 1179 (1997); E. Runge and R. Zimmermann, Phys. Status Solidi B 221, 269 (2000).

[11] I. Carusotto and C. Ciuti, Phys. Rev. Lett. 93, 166401 (2004); C. Ciuti and I. Carusotto, Phys. Status Solidi B 242, 2224 (2005).

[12] P. R. Eastham and P. B. Littlewood, Solid State Commun. 116, 357 (2000); Phys. Rev. B 64, 235101 (2001).

[13] D. M. Whittaker, Phys. Rev. Lett. 80, 4791 (1998).

[14] D. M. Whittaker, Phys. Rev. B 61, R2433 (2000).

[15] A. V. Shchegrov et al., Phys. Rev. Lett. 84, 3478 (2000).

[16] M. Gurioli et al., Phys. Rev. Lett. 94, 183901 (2005). 